\title{
The Usefulness of CytoFoam-Core in the Diagnosis of Thyroid Metastasis From Colon Cancer: A Case Report
}

\author{
Silvia Taccogna ${ }^{1, *}$, Sara Ienzi ${ }^{1}$, Stefano Amendola ${ }^{2}$, Claudio Ventura ${ }^{2}$, \\ Elena Chiapparino³, Martina D'Angelo', Basilio Lippi ${ }^{4}$, Margherita \\ Guarino $^{5}$ \\ ${ }^{1}$ Anatomic Pathology Unit, Regina Apostolorum Hospital, Albano Laziale, \\ Rome, Italy \\ ${ }^{2}$ Section of Endocrinology and Diabetology, Jewish Hospital, Rome, Italy \\ ${ }^{3}$ Division of Medical Oncology, 'S. Giovanni Evangelista' Hospital, Tivoli, \\ Rome, Italy \\ ${ }^{4}$ Diagnostic Unit, Jewish Hospital, Rome, Italy \\ ${ }^{5}$ Diagnostic Laboratory Unit, Jewish Hospital, Rome, Italy \\ *Corresponding author: Silvia Taccogna, Anatomic Pathology, Regina \\ Apostolorum Hospital Via S. Francesco 50, 00041 Albano Laziale, Rome, \\ Italy. Tel: +390693298423; Fax: +39069321138; E-mail: staccogna@, \\ reginaapostolorum.it
}

DOI: $10.30699 /$ mci.4.4.447

Submitted: 15 August 2020

Revised: 7 September 2020

Accepted: 23 September 2020

e-Published: 1 October 2020

Keywords:

Colon

Carcinoma

Thyroid Gland
Introduction: We report a case of a 64-year old man with a history of colon carcinoma. $\mathrm{He}$ underwent fine-needle aspiration by CytoFoam-core and diagnosis of metastatic colon cancer to the thyroid gland.

Case presentation: In this case report, a solid thyroid nodule in the right lobe of a 64-year-old male was aspirated. The cytological smears showed a small group of atypical cells with the obvious nucleolus, necrosis, and atypical tall columnar cells. Since the patient had undergone surgery for rectal cancer at age 63, suspicion of metastasis from colon cancer was raised. This was corroborated by CytoFoam-core immunocytochemistry; showing a positive staining pattern for caudal type homeobox transcription factor $2(\mathrm{~cd} \times 2)$ and cytokeratin 20 (CK20); while thyroglobulin (TG) and thyroid transcription factor-1 (TTF-1) were both negative.

Conclusion: The main objective was to correctly identify the metastatic thyroid carcinoma (MTC) and exclude other clinical entities as primary diseases of the thyroid gland. Cytology plays a crucial role in the follow-up and the therapeutic management of the patients. The CytoFoam-core preparations were diagnostic of metastatic colon cancer to the thyroid gland. In this context, the CytoFoam-core could be of great help, especially in previous oncologic history cases.

(C) 2020. Multidisciplinary Cancer Investigation

\section{INTRODUCTION}

Secondary tumors of the thyroid gland are uncommon as the frequency of metastasis accounts for $0.1-0.3 \%$ of thyroid malignancies [1]. Secondary tumors may involve the thyroid gland as a result of direct extension from contiguous structures or as metastatic foci from a distant primary malignancy. An accurate and timely diagnostic definition of these cases is essential as a confirmed metastatic disease has crucial implications for clinical management and prognosis of the primary malignancy $[2,3]$. Lungs 
are the most common primary lesions, followed by kidney, and gastrointestinal system malignancies. Breast, uterus, larynx, and trachea are less frequent primary sources. The interval from the diagnosis of the primary tumor to the identification of thyroid metastasis is reported to vary from 1 month to 16 years [4]. The patients with colorectal cancer in $30-40 \%$ of cases show advanced locoregional or distant disease at the initial diagnosis, that cannot be cured with surgery alone [4]. Regional lymph nodes, liver, lung, and peritoneum are the most common sites for colon cancer metastasis. Herein, we report a case of colonic adenocarcinoma metastasis to the thyroid gland, right adrenal gland, and lung in absence of liver involvement. While fine-needle aspiration biopsy (FNAB) under ultrasound (US) guidance provided a Bethesda V cytology, suspicious for malignancy, while the CytoFoamcore correctly suggested the diagnosis of metastatic colonic carcinoma. The patient received palliative radiotherapy on the neck and supportive care. His decease occurred in June 2018.

\section{CASE PRESENTATION}

We report a case of a 64-year old man referred to the Department of Diabetology for an asymptomatic thyroid nodule. His previous medical history revealed that he underwent radical surgery for rectal cancer one year ago. The pathology report showed a moderately differentiated adenocarcinoma (G2) with no lymphatic and venous invasion. TNM classification was pT3 pN0 M0, stage IIB, according to the international union against cancer tumor-node-metastasis 7 th classification. Computer tomography (CT) examination did not reveal distant metastasis. The patient was disease-free for one year, but in May 2016 a routine CT revealed lesions in the thyroid, lung, and right adrenal. A thyroid nodule was found in the right lobe as a hard, fixed, and non-tender mass, measuring approximately $45 \mathrm{~mm}$ in its major diameter. The surrounding thyroid gland was normal, but US examination revealed the presence of cervical and supraclavicular lymphadenopathy. The nodule was hypoechoic, with heterogeneous structure, irregular margins, and scanty intra-nodular vascular signals without evidence of extracapsular spread (Figure $1 \mathrm{~A}$ and $1 \mathrm{~B})$.
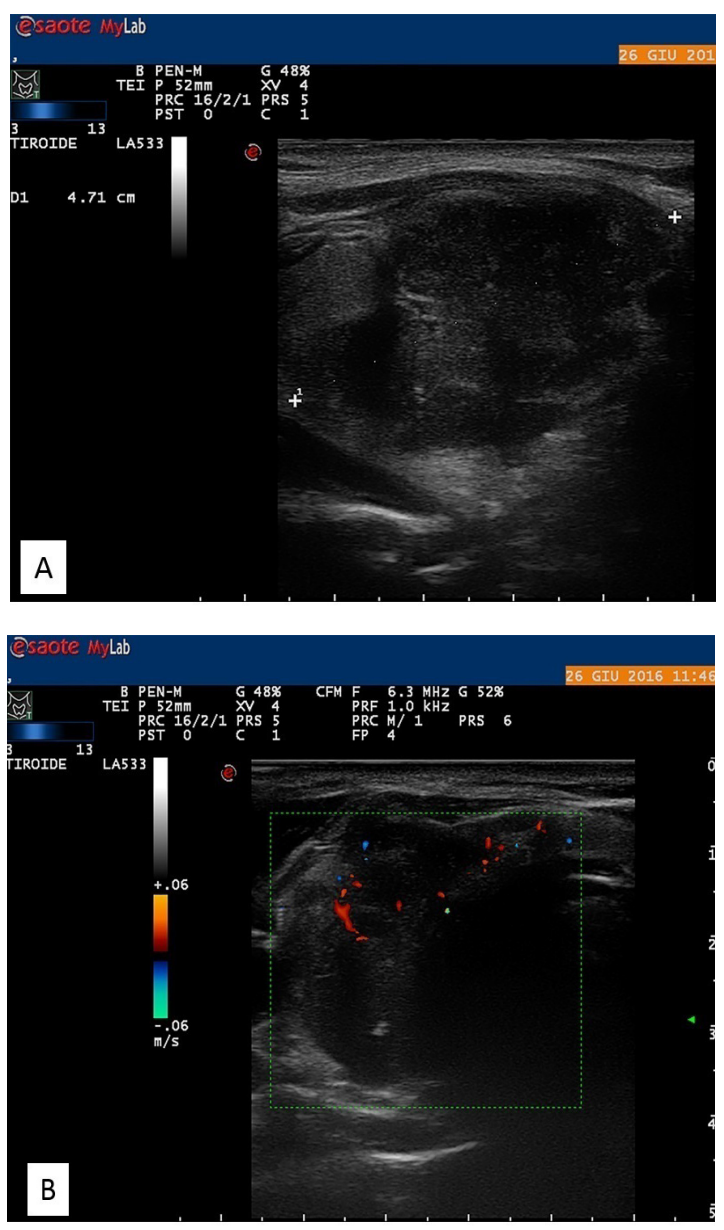

Figure 1: Ultrasound Scan of the Right Thyroid Lobe

(A) Deeply hypoechoic nodule, $47 \times 40 \times 35 \mathrm{~mm}$, with heterogeneous structure and irregular margins. (B) Colordoppler evaluation demonstrates scanty intra-nodular vascular images. No sonographic evidence of thyroid capsule infiltration or of pathologic adenopathy is present.

The patient was referred for US-guided FNAB of the thyroid lesion. The cytological smears showed a small group of atypical cells with obvious nucleolus (Figure 2).

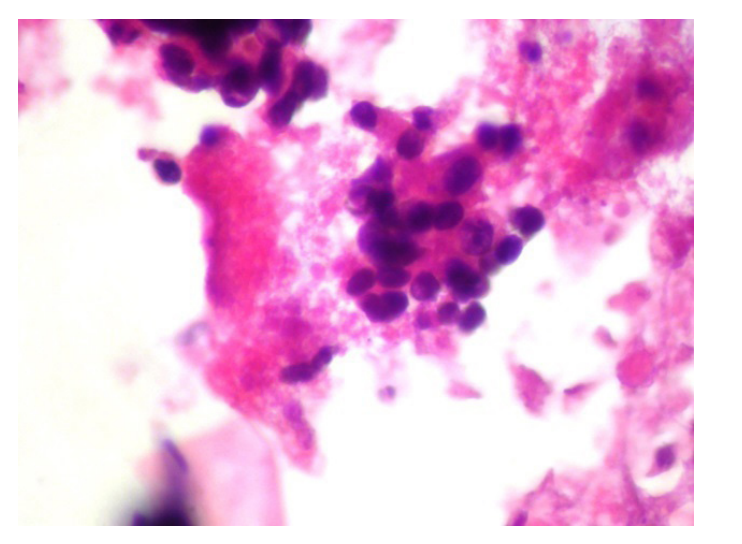

Figure 2: Metastasis of Colon Cancer to the Thyroid Gland Cytological evidence of a malignant epithelial cell population; the neoplastic cells are note aggregated into groups with nucleolus evident (papanicolaou staining $\times 400$ ). 
No papillary carcinoma cytologic features were identified and the lesion was diagnosed as Bethesda $\mathrm{V}$ (suspicious for malignancy) [5]. The previous diagnosis raised the clinical suspicion of a potential metastasis from colon cancer. A second cytology sampling, performed with the use of a modified $21 \mathrm{G}$ needle named CytoFoam core (Exmoor Innovations, Bio-Optica Italian distributor), was performed to rule out the presence of an anaplastic (undifferentiated) primary thyroid carcinoma and to confirm the clinical suspicion of a metastasis from the primary colonic neoplasm [6]. The hematoxylineosin staining performed on a formalin-fixed sponge support, revealed atypical groups of cohesive columnar cells with increased nucleus-cytoplasm ratios in a background of necrotic debris. The nuclei were large, elongated and palisading with evident nucleolus (Figure 3).

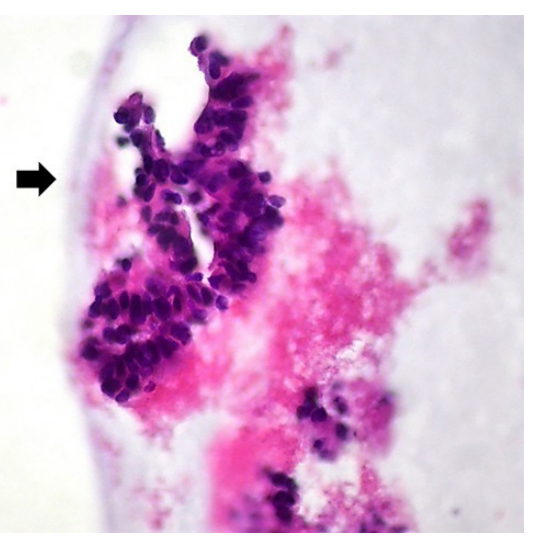

Figure 3: CytoFoam-Core From Fine-Needle Aspiration Sampling (23 Gauge Needle)

Hematoxylin \& Eosin staining (H\&E) of the neoplastic aggregates entrapped in the foam structure (arrow) $(\times 400)$

The neoplastic cells are diffusely and strongly immunoreactive for cytokeratin 20 (Figure 4A). TTF1 and TG were negative in neoplastic cells and positive in thyroid cells (Figure 4B). The immunostaining for $\mathrm{cd} \times 2$ was positive in the neoplastic cells and resulted as negative in thyrocytes (Figure 4C and 4D).

As a whole, the cytological findings were diagnostic of metastatic colon cancer to the thyroid gland. The material was also evaluated for kirsten rat sarcoma (KRAS) and neuroblastoma rat sarcoma (NRAS) molecular assay. Accordingly, NRAS is considered as the wild type but KRAS codon12-mutation ( $p$ Gly12Asp (c.35G $>$ A) represents the primary tumor. From June to September 2016 the patient received chemotherapy and anti-angiogenesis of Avastin (bevacizumab).

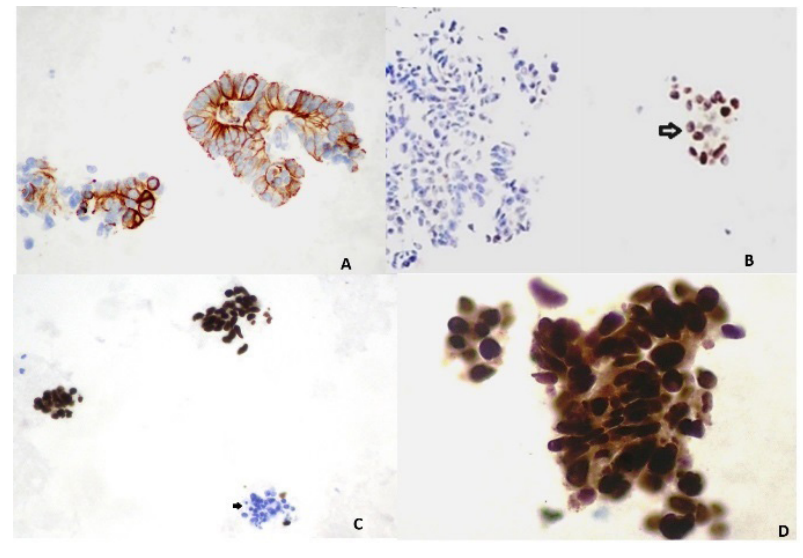

Figure 4: Immunohistochemistry

(A) The cells express strong cytoplasmic CK 20 positivity $(\times 200)$; (B) The complete absence of TTF1 staining in the neoplastic cells; note the positivity (empty arrow) of the thyrocytes $(\times 400)$; (C) The neoplastic cells are positive per $\mathrm{cd} \times 2$, the thyrocytes are negative (arrow) $(\times 200)$; (D) note the intense nuclear positivity at higher magnification $(\times 400)$.

The US showed a reduction of the dimension of adrenal and thyroid nodules in September 2016, as a satisfactory response. In April 2017, the total body $\mathrm{CT}$ demonstrated a progression. The patient received palliative radiotherapy on the neck and supportive care. His decease occurred in June 2018.

\section{DISCUSSION}

The incidence of intrathyroidal metastasis is low [3] and varies from 1.25 to $24.0 \%$ in autopsy series of cancer patients [7]. The gastrointestinal malignancies rarely metastasize into the thyroid gland; the colon and the rectum are the main origin [8]. Even if the thyroid is one of the most perfused organs in the body, it is rarely considered as a solitary site of metastases that are usually asymptomatic [79]. However, in patients with a history of cancer and suspicious thyroid nodules the possibility of a metastasis should be considered. As primary thyroid carcinomas are usually low-grade neoplasms, the presence of frank atypia in the cytological sample should raise the suspicion of a metastatic carcinoma [3].The presence of thyroid invasion does not negatively impact the overall survival and the occurrence of thyroid metastasis influence the prognosis as the metastasis to other organs. So, the overall survival depends on the prognosis of the primary tumor and of the metastatic state $[7,8]$. The 
management of thyroid nodules is largely directed by the cytologic findings from FNAB, which represents the gold standard for diagnosis of nodular lesions; however, these results must be considered in the context of US and clinical findings [10]. The imaging can be helpful in the diagnostic workup of these cases, but the radiological findings alone are usually nonspecific and cannot be used conclusively to distinguish primary from secondary lesions [11]. Cytology plays a key role in the diagnostic workup of metastatic neoplasms to the thyroid [12] but its main limitation is the impossibility to use part of the sampled material for ancillary studies [2]. Immunohistochemistry (IHC) and other molecular studies are often required to fully characterize tumors diagnosed by FNAB. These supplementary investigations are best performed on cell blocks or CytoFoam-core support. While the cell blocks are time-consuming and sometimes subjected to loss of cellular material during processing, the CytoFoam-core sample is a diagnostic tool for both IHC and molecular analysis [13, 14]. A single passage provides material for a cell-block due to the presence of the adsorbent support inserted in a 21 -gauge needle on which the cells remain trapped. The specimen obtained from the CytoFoam-core is processed as a tissue fragment, formalin-fixed, and paraffin-embedded sample [6]. So, the use of a device that collects the material during the FNA procedure reduces laboratory workflow time while improving the quality of the cytological sample. The availability of additional material, accessible without more invasive procedures such as coreneedle biopsy, allows a complete morphological, immunohistochemical and molecular evaluation.

Secondary malignancies of the thyroid gland are a rare entity and colon metastasis are even rarer, despite the high prevalence of colorectal cancer (CMT) in developed countries. The diagnosis of distant metastatic disease has become more frequent, including its appearance in infrequent sites as the thyroid gland, due also to the improvement in diagnostic techniques. The CytoFoam-core sample plays a pivotal role to correctly identify the presence of CMT, excluding alternative clinical entities as primary disease of the thyroid gland, and allows the use of molecular assays for followup and therapeutic management of the patients. CytoFoam-core may be used as a reliable diagnostic tool for cytological assessment of thyroid nodules, especially in case of uncertain cytological diagnosis and previous oncologic history.

\section{ACKNOWLEDGEMENTS}

The authors would like to thank Antonio Cerrito for his assistance with the preparation of the images and Massimo Pigliapoco for the English revision.

\section{CONFLICTS OF INTEREST}

The authors declared no conflict of interest.

\section{ETHICS APPROVAL}

Informed consent was obtained from the patient's wife to publish this case report in a medical journal.

\section{REFERENCES}

1. Zhang L, Liu Y, Li X, Gao W, Zheng C. Metastases to the thyroid gland: A report of 32 cases in PUMCH. Medicine (Baltimore). 2017;96(36):e7927. DOI: 10.1097/ MD.0000000000007927 PMID: 28885347.

2. Pastorello RG, Saieg MA. Metastases to the Thyroid: Potential Cytologic Mimics of Primary Thyroid Neoplasms. Arch Pathol Lab Med. 2019;143(3):394-9. DOI: 10.5858/ arpa.2017-0570-RS PMID: 30444438.

3. Fadda G, DeLellis RA, Sadow P. Tumours of the Thyroid Gland. In: Lloyd RV, Osamura RY, Klöppel G, Rosai J, editors. WHO Classification of Tumours of Endocrine Organs. 10. 4th ed. Lyon, France: International Agency for Research on Cancer (IARC); 2017. p. 142-3.

4. Ferlay J, Colombet M, Soerjomataram I, Mathers C, Parkin DM, Pineros M, et al. Estimating the global cancer incidence and mortality in 2018: GLOBOCAN sources and methods. Int J Cancer. 2019;144(8):1941-53. DOI: 10.1002/ijc.31937 PMID: 30350310.

5. Cibas ES, Ali SZ. The 2017 Bethesda System for Reporting Thyroid Cytopathology. Thyroid. 2017;27(11):1341-6. DOI: 10.1089/thy.2017.0500 PMID: 29091573.

6. Mayall FG. An FNA cytology foam core device for making cell blocks. J Clin Pathol. 2012;65(10):959-61. DOI: 10.1136/jclinpath-2012-200858 PMID: 22685261.

7. Nixon IJ, Coca-Pelaz A, Kaleva AI, Triantafyllou A, Angelos P, Owen RP, et al. Metastasis to the Thyroid Gland: A Critical Review. Ann Surg Oncol. 2017;24(6):1533-9. DOI: 10.1245/s10434-016-5683-4 PMID: 27873099.

8. Amante MA, Real IO, Bermudez G. Thyroid metastasis from rectal adenocarcinoma. BMJ Case Rep. 2018;2018. DOI: $10.1136 /$ bcr-2018-225549 PMID: 30108119.

9. Chen H, Nicol TL, Udelsman R. Clinically significant, isolated metastatic disease to the thyroid gland. World J Surg. 1999;23(2):177-80; discussion 81. DOI: $10.1007 /$ p100013162 PMID: 9880428 .

10. Haugen BR, Alexander EK, Bible KC, Doherty GM, Mandel SJ, Nikiforov YE, et al. 2015 American Thyroid As- 
sociation Management Guidelines for Adult Patients with Thyroid Nodules and Differentiated Thyroid Cancer: The American Thyroid Association Guidelines Task Force on Thyroid Nodules and Differentiated Thyroid Cancer. Thyroid. 2016;26(1):1-133. DOI: 10.1089/thy.2015.0020 PMID: 26462967.

11. Kim HK, Kim SS, Oak CY, Kim SJ, Yoon JH, Kang HC. Diffuse metastasis to the thyroid: unique ultrasonographic finding and clinical correlation. J Korean Med Sci. 2014;29(6):818-24. DOI: 10.3346/jkms.2014.29.6.818 PMID: 24932084.

12. Kim TY, Kim WB, Gong G, Hong SJ, Shong YK. Metastasis to the thyroid diagnosed by fine-needle aspiration biopsy. Clin Endocrinol (Oxf). 2005;62(2):236-41. DOI: 10.1111/j.1365-2265.2005.02206.x PMID: 15670202.

13. Erra S, Lancella A, Rotella J, Ricotta S, Mazzoni E, Carlon E. A Rare Case of Neck Metastasis from a Thyroid Papillary Carcinoma After Seventeen Years Diagnosed with US-Guided Fine Needle Aspiration Cytology. Int J Clin Oncol Cancer Res. 2017;2(5):106-9. DOI: 10.11648/j.ijcocr.20170205.12.

14. Taccogna S, Guglielmi R, Persichetti A, Morano C, Angelini $\mathrm{F}$, Ienzi $\mathrm{S}$, et al. Carcinomas of the Thyroid with Ewing Family Tumor Elements (CEFTEs): A Diagnostic Challenge Before Surgery. Head Neck Pathol. 2020. DOI: 10.1007/s12105-020-01145-Z PMID: 32077055. 